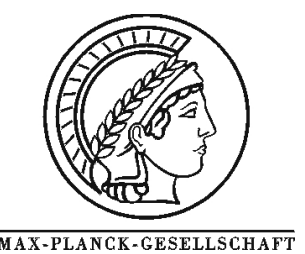

Nuclear Instruments and Methods in Physics Research A, 470 (2001), 302-305

\title{
Combined Application of XANES and XPS to Study Oxygen Species Adsorbed on Ag Foil
}

\author{
V.I. Bukhtiyarov ${ }^{*}$, M. Hävecker ${ }^{2}$, V.V. Kaichev ${ }^{1}$, A. Knop-Gericke², R.W. Mayer ${ }^{2}$, R. Schlögl ${ }^{2}$ \\ ${ }^{1}$ Boreskov Institute of Catalysis, Lavrentieva prosp., 5, 630090, Novosibirsk, Russia \\ ${ }^{2}$ Department of Inorganic Chemistry, Fritz-Haber-Institute of the MPG, Faradayweg 4-6, 14195 Berlin, Germany \\ ${ }^{*}$ Corresponding author: e-mail vib@ctalysis.nsk.su, phone +7 38323437 67, fax +7 3832343056
}

Keywords: Silver, oxygen, adsorption, XANES, XPS

Studying of gas interaction with a solid is of great significance for the field of heterogeneous catalysis where activation of gas phase reagents is first step of catalytic process. In the few past years X-ray absorption near edge structure spectroscopy (XANES) has been proven to be an important tool for this purpose. Being an element-specific method, XANES provides direct information about the unoccupied electronic states of the element analyzed [1]. These valence orbitals reflect the nature of adspecies-metal bonding, and can participate into the formation of new chemical bonds in the course of catalytic reactions. Other advantage of XANES is the possibility of in-situ measurement [2]. In spite of so evident perspectives the examples of X-ray absorption studies of the adsorbed species are quite rear. One of the reasons responsible for such situation is necessity to use an UHV installation attached to Synchrotron Radiation Source. It allows one to clean the sample from undesired impurities, which could contribute to total XAS spectrum, and to adsorb the gases at controlled conditions. Furthermore, to be sure in the assignment of the XAS spectra to any adsorbed species, which in principle can result in few features, the researchers should provide the characterization of the surface by methods sensitive to the chemical compositions of the adlayers analyzed.

In the present paper devoted to study oxygen species realized in the course of ethylene epoxidation over silver, we took into account both limitations. The photonenergy-dependent XAS measurements were performed in a double-chamber UHV system with a base pressure of $<5 \times$ $10^{-9}$ mbar attached to the HE-TGM 1 beamline at the Berliner synchrotron radiation source BESSY-I [3]. The composition of oxygen overlayers on surface of the same polycrystalline silver foil was characterized using a VG ESCALAB High Pressure X-ray photoelectron spectrometer [4]. The choice of the silver as object for this study has been defined by its industrial importance as the unique heterogeneous catalyst for ethylene epoxidation [5]. The sample was cleaned by the standard high vacuum cycle: $\mathrm{Ar}^{+}$sputtering, annealing in 10 mbar of $\mathrm{O}_{2}$ at $570 \mathrm{~K}$ and flashing up to 800 $\mathrm{K}$ in UHV.

Figure 1 shows $\mathrm{O} K$-edge XAS and O1s core level spectra recorded after treatment of the silver by $\mathrm{C}_{2} \mathrm{H}_{4}(2 \%)+\mathrm{O}_{2}$ reaction mixture for 6 hours at $T=470$ $\mathrm{K}$ and $P=2$ mbar followed by step-wise heating of the pre-treated surface in UHV. To observe the XAS signals from adsorbed species, the raw spectra were divided by the spectrum of a clean silver surface. It is well-known [6] that these conditions provide the activation of clean silver surface for epoxidation due to accumulation of the adsorbed oxygen epoxidizing ethylene to ethylene oxide [4,7]. This species was called as electrophilic oxygen [4,6-7]. O1s spectra presented in the fig. $1 \mathrm{~b}$ is in agreement with the previ- 
ous data: O1s feature (fig. $1 \mathrm{~b}$, curve 1) at 530.4 indicates the formation of the electrophilic oxygen.


Fig.1. O $K$-edge X-ray absorption (a) and O1s core level photoemission (b) spectra from the silver foil pre-treated for 6 hours by $\mathrm{C}_{2} \mathrm{H}_{4}(2 \%)+\mathrm{O}_{2}$ reaction mixture at $T=470 \mathrm{~K}$ and $P=2$ mbar (1) followed by stepwise heating in UHV at $T=570 \mathrm{~K}(\mathbf{2})$ and $770 \mathrm{~K}(\mathbf{3})$.

The pre-treated silver surface is characterized by XAS spectrum that can be divided into two regions: a weak signal near threshold ( $\sim 531 \mathrm{eV}$ photon energy) and a broad one at 5-20 eV above the threshold (fig. 1a, curve 1). However, behavior of these signals at sample heating is quite different determining their assignment to different oxygen species. Removal of the signal at $\sim 531 \mathrm{eV}$ accompanied also by disappearance of part of the higher photon energy signal (fig.1a, difference curve 1-2) occurs at $570 \mathrm{~K}$, while the rest broad XAS signal disappears after heating up to 770 $\mathrm{K}$. Heating at $770 \mathrm{~K}$ causes also disappearance of the $\mathrm{O} 1 \mathrm{~s}$ feature at $530.4 \mathrm{eV}$. The latter fact leads us to assignment of the broad $\mathrm{O} K$-edge feature at $\sim 541 \mathrm{eV}$ to the electrophilic oxygen [8].

The XAS signals at $\sim 531$ and $\sim 539 \mathrm{eV}$ may be due to the nucleophilic oxygen since their removal temperature coincides with the desorption temperature of this oxidelike oxygen [4-5,7-9]. To check this suggestion we have provided comparative analysis of $\mathrm{O} K$-edge and $\mathrm{O} 1 \mathrm{~s}$ spectra monitored during $\mathrm{O}_{2}$ adsorption at $P=10^{-4}$ and $T=470 \mathrm{~K}$, i.e. at the conditions when the structure of surface silver oxide is effectively formed [4]. The possibility of in-situ measurements both of XAS and of XPS spectra is of great importance, since these conditions provide steady-state saturation $(\Theta=0.5)$ of the silver surface by this highly reactive oxygen. The corresponding spectra are shown in fig.2.

One can see that in full agreement with our expectations, $\mathrm{O}_{2}$ adsorption gives rise to $\mathrm{O} 1 \mathrm{~s}$ feature with binding energy $(528.3 \mathrm{eV})$ typical of the nucleophilic oxygen (fig.2b, curve 1). This is accompanied by an appearance of two features in XANES oxygen spectrum at $\sim 531$ and $\sim 539 \mathrm{eV}$ (fig.2a, curve 1). Comparison of this spectrum with the difference one taken from the fig.1a shows their great similarity indicating that both spectra belong to the nucleophilic oxygen. This conclusion is confirmed by full disappearance of the corresponding signals from $\mathrm{O} K$-edge and $\mathrm{O} 1 \mathrm{~s}$ spectra as the sample is annealed at $570 \mathrm{~K}$.
Thus, namely the nucleophilic oxygen is registered together with the electrophilic one in ex-situ XAS spectra of the silver foil pre-treated by $\mathrm{C}_{2} \mathrm{H}_{4}+\mathrm{O}_{2}$ reaction mixture.
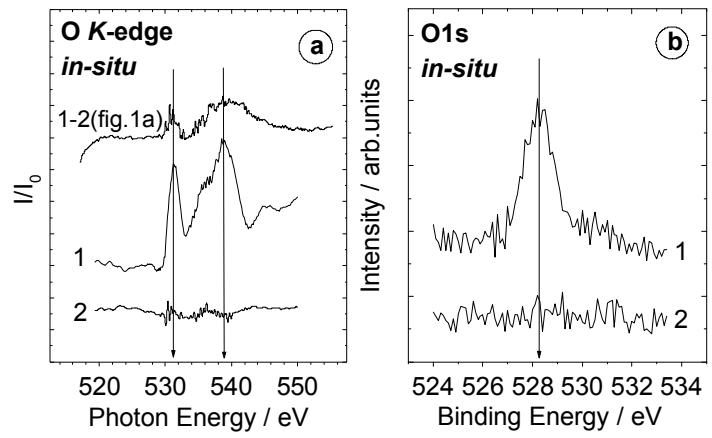

Fig.2. O $K$-edge X-ray absorption (a) and O1s core level photoemission (b) spectra from the silver foil during $\mathrm{O}_{2}$ adsorption at $T=470 \mathrm{~K}$ and $P=10^{-4} \mathrm{mbar}$ (1) followed by stepwise heating in UHV at $T=570 \mathrm{~K}$ (2).

The absence of the corresponding feature at 528.3 $\mathrm{eV}$ in $\mathrm{O} 1 \mathrm{~s}$ spectra of the pre-treated surface (fig. $1 \mathrm{~b}$, curve 1) is explained by better vacuum conditions of the XAS spectrometer (oil-less turbovac pumping) compared to the XPS spectrometer. The use of polyphenol ethers as a working liquid in diffusion pumps of the XPS spectrometer can cause the removal of this highly active species due to titration by hydrocarbon background. This fact indicates that it would be better to carry out XAS and XPS measurements in one UHV installation.

Nevertheless, the comparison of the XANES and XPS spectra carried out for all experiments presented in this paper allows us to select the observed XAS features and to assign them to the electrophilic and nucleophilic oxygen. This is of great importance for catalysis, since namely these oxygen species have been suggested to make up the surface centers to be responsible for the formation of ethylene oxide $[4,7]$. The electrophilic oxygen is characterized by broad feature at $\sim 541 \mathrm{eV}$, while two $\mathrm{O} K$-edge features at $531 \mathrm{eV}$ and $\sim 539 \mathrm{eV}$ are characteristic of the nucleophilic one, i.e. the obtained XAS data indicate different character of oxygen-silver bonding for these catalytically interesting oxygen species.

According to literature data [10-11], the range of photon energy near threshold $(\sim 531 \mathrm{eV})$ is attributed to oxygen $\mathrm{p}$ orbitals hybridized with silver $4 \mathrm{~d}$ character, while the signals in the region of $5-20 \mathrm{eV}$ above threshold originates from oxygen $\mathrm{p}$ character hybridized with metal 5sp states. Then, one can say that the electrophilic oxygen exhibits mainly hybridization with $5 \mathrm{sp}$ silver orbitals, whereas $\mathrm{Ag} 4 \mathrm{~d}$ orbitals contribute considerably to O-Ag bond for the nucleophilic oxygen. Obviously, these variations should be taken into account for explanation of differences in O1s binding energy values for these oxygen species. Another conclusion following from the XAS data concerns with the high covalence of the silver-oxygen bond for both oxygen species. Indeed, in a purely ionic model, oxygen would have the configuration $\mathrm{O} 1 \mathrm{~s}^{2} 2 \mathrm{~s}^{2} 2 \mathrm{p}^{6}$ and the $1 \mathrm{~s} \rightarrow 2 \mathrm{p}$ channel would 
be closed for XAS. Covalence reduces the number of filled states with $\mathrm{O} 2 \mathrm{p}$ character, so that the strength of the $\mathrm{O} K$ edge signal is related to the degree of covalence [11].

\section{Acknowledgements}

This work was supported in part by Russian Foundation for Basic Research, grant No. 00-15-99335. The authors would like to thank BESSY staff for the help in carrying out the XAS experiments. Furthermore, V.I.B. gratefully acknowledges the Max-PlanckGesselschaft for financial support of his visit to FHI and work on BESSY.

\section{References}

[1] J. Stöhr, in: R. Gomer (Ed.) NEXAFS Spectroscopy, Springer Series in Surface Sciences, vol. 25, (Springer, Heidelberg, 1992).

[2] M. Hävecker, A. Knop-Gericke, Th. Schedel-Niedrig, R Schlögl, Angew. Chem. Int. Ed. 37 (1998) 1939.

[3] A. Knop-Gericke, M. Hävecker, Th. Schedel-Niedrig, Nucl. Instrum. Meth. Phys. Resear. A 406 (1998) 311.

[4] V.I. Bukhtiyarov, A.I.Boronin, I.P. Prosvirin, V.I. Savchenko, J. Catal. 150 (1994) 262.

[5] R.A. van Santen, H.P.C.E. Kuipers, Adv. Catal. 35 (1987) 26.

[6] R.B. Grant, R.M. Lambert, J. Catal. 92 (1985) 364.

[7] V.I. Bukhtiyarov, I.P. Prosvirin, R.I. Kvon, Surf. Sci. 320 (1994) L47.

[8] V.I. Bukhtiyarov, V.V.Kaichev, I.P. Prosvirin and E.A. Podgornov, Catal. Lett., 1999, 57, 233.

[9] C. T. Campbell and M. T. Paffett, Surf. Sci. 143 (1984) 517.

[10] F.M.F. de Groot, M. Grioni, J.C. Fuggle, J. Ghijsen, G.A. Sawatzky, H. Petersen, Phys. Rev. B 40 (1989) 5715.

[11] Th. Shedel-Niedrig, X. Bao, M. Muhler, R. Schlögl, Ber. Bunsengen. Phys. Chem. 101 (1997) 994. 